Yoshimura, $\mathrm{H}$.

Osaka J. Math.

28 (1991), 285-294

\title{
ON FINITELY PSEUDO-FROBENIUS RINGS
}

\author{
HIROSHI YOSHIMURA
}

(Received April 19, 1990)

In this paper we are concerned with FPF rings and GFC rings. In section 2 we provide some results about these rings; we show that every right GFC ring is essentially bounded (Proposition 4) and give a characterization of right FPF rings (Theorem 11). Finally, we present examples to illustrate Theorem 11.

\section{Preliminaries}

Throughout this paper $R$ will always denote an associative ring with identity and all $R$-modules will be unital.

If every finitely generated faithful right $R$-module is a generator of the category mod- $R$ of right $R$-modules then $R$ is said to be right finitely pseudoFrobenius (right FPF). Following [2], $R$ is said to be generated by faithful cyclic (right GFC) if every faithful cyclic right $R$-module is a generator of mod-R. Right FPF rings are obviously right GFC and the class of right FPF rings includes right $\mathrm{PF}$ rings and Dedekind domains.

Let $M$ be a right $R$-module, $X$ (resp. $S$ ) a subset of $M$ (resp. $R), A$ a right ideal of $R$ and $n$ a positive integer. Then we denote by $r_{R}(X)\left(\operatorname{resp} . l_{R}(S)\right)$ the right (resp. left) annihilator of $M$ (resp. $S$ ) in $R$, by $\operatorname{Tr}_{R}(M)$ the trace ideal of $M$, i.e., $\operatorname{Tr}_{R}(M)=\Sigma\left\{\operatorname{Im}(f) \mid f \in \operatorname{Hom}_{R}(M, R)\right\}$ and by $Z_{r}(M)$ the singular submodule of $M$, i.e., $Z_{r}(M)=\left\{x \in M \mid r_{R}(x)\right.$ is essential in $\left.R_{R}\right\}$. Further we denote by $M^{(n)}$ the direct sum of $n$ copies of $M$. By ideals we will mean two-sided ideals of $R$.

Let $\tau$ be a hereditary torsion theory for mod-R. Then we denote by $L(\tau)$ the Gabriel topology corresponding to $\tau$ and by $\tau(M)$ the $\tau$-torsion submodule of $M$. Set $B / A=\tau(R / A)$. If $A$ is an ideal of $R$ then we see that $B$ becomes an ideal; hence in particular, $\tau(R)$ is an ideal of $R$. A submodule $N$ of $M$ is $\tau$-closed in $M$ if $M / N$ is $\tau$-torsionfree. We let $G$ denote the Goldie torsion theory for mod- $R$. We then note that $M$ is $G$-torsionfree if and only if $Z_{r}(M)=$ 0 , i.e., $M$ is right non-singular.

We refer to [8] for all the torsion-theoretic notions used in this paper.

The following easy result will be used repeatedly without reference throughout the sequel. 
Lemma. For a right ideal $A$ of $R, \operatorname{Tr}_{R}(R / A)=l_{R}(A) R$.

\section{FPF (GFC) rings}

A submodule $N$ of a right $R$-module $M$ is essentially closed in $M$ if it has no proper essential extensions inside $M$, or equivalently there exists a submodule $L$ of $M$ such that $N$ is maximal with respect to $N \cap L=0$. We note that every $G$-closed submodule of $M$ is essentially closed in it. Further, it is easy to show that if $L \leqq N \leqq M$ are right $R$-modules such that $L$ is essentially closed in $M$ and $N$ is essential in $M$ then $N / L$ is essential in $M / L$.

Now, the following result is easy.

Lemma 1. An ideal $I$ of $R$ is $G$-closed in $R_{R}$ if and only if it is essentially closed in $R_{R}$ and $R / I$ is right non-singular over $R / I$.

Lemma 2. Let $I$ be an ideal of $R$ and $A$ a right ideal of $R$ such that $I+A$ is essential in $R$. If $R / A$ is a generator of mod-R then $I$ is essential in $R_{R}$.

Proof. Assume that $R / A$ is a generator of $\bmod -R$, that is, $l_{R}(A) R=R$. Then there exists a finite number of elements $a_{i} \in l_{R}(A)$ and $b_{i} \in R(i=1, \cdots, n)$ such that $1=\sum_{i=1}^{n} a_{i} b_{i}$. Setting $B=\left\{x \in R \mid b_{i} x \in I+A\right.$ for all $\left.i=1, \cdots, n\right\}$, we see from the essentiality of $I+A$ that $B$ is an essential right ideal of $R$. It then follows that $I$ is essential in $R_{R}$, because $B \leqq I$.

The following result shows that if $R$ is right GFC then $Z_{r}(R)$ contains all nilpotent one-sided ideals of $R$.

Proposition 3. Assume that $R$ is right GFC, and let $A$ be a nilpotent right ideal of $R$. Then $r_{R}(A)$ is essential in $R_{R}$.

Proof. Let $n$ be the nilpotent index of $A$. The assertion is clear for $n=1$.

Now let $n>1$ and assume that the assertion is true for every nilpotent right ideal of $R$ with nilpotent index $n^{\prime}<n$. Choose a right ideal $B$ of $R$ maximal with respect to $B \leqq r_{R}\left(A^{2}\right)$ and $B \cap r_{R}(A)=0$. Then $B \oplus r_{R}(A)$ is essential in $r_{R}\left(A^{2}\right)$. Since $A^{2}$ has nilpotent index $\leqq n-1$, the induction hypothesis assures that $r_{R}\left(A^{2}\right)$ is essential in $R_{R}$. Thus $B \oplus r_{R}(A)$ is essential in $R$. On the other hand, we have $A r_{R}(R / B) \leqq B \cap r_{R}(A)=0$; hence $r_{R}(R / B) \leqq B \cap r_{R}(A)=0$. Since $R$ is right GFC, $R / B$ is a generator of mod-R. It now follows from Lemma 2 that $r_{R}(A)$ is essential in $R_{R}$.

If every essential right ideal of $R$ contains an ideal essential in $R$ as a right ideal then $R$ is said to be right essentially bounded. By [3, Proposition 1.3B], every essential right ideal of a right FPF ring contains a non-zero ideal. On the other hand, by [4, Corollary 2.2.], a left Noetherian, right FPF and right order 
in a $Q F$ ring is right essentially bounded. However, we see that every right GFC ring is right essentially bounded. To show this, let $A$ be an essential right ideal of a right GFC ring $R$, and choose a right ideal $B$ of $R$ maximal with respect to $B \leqq A$ and $r_{R}(R / A) \cap B=0$. We then see that $r_{R}(R / A) \oplus B$ is essential in $R$, and further that $R / B$ is faithful; hence it is a generator of mod-R. Now Lemma 2 shows that $r_{R}(R / A)$ is essential in $R_{R}$, as desired. Thus we have the following result.

Proposition 4. Every right GFC ring is right essentially bounded.

From the above two Propositions, we obtain the following result.

Corollary 5. Assume that $R$ is right GFC. Then an ideal $I$ of $R$ is $G$ closed in $R_{R}$ if and only if it is a semiprime ideal which is essentially closed in $R_{R}$.

Proof. Assume that $I$ is $G$-closed in $R_{R}$. To show that $I$ is a semiprime ideal of $R$, let $J$ be an ideal of $R$ such that $I \leqq J$ and $J^{2} \leqq I$. Choose a right ideal $A$ of $R$ such that $A \leqq J$ and $A \cap I=0$. Since $R / I$ is a non-singular right $R$ module, so is $A$. On the other hand, $A^{2} \leqq A \cap J^{2} \leqq A \cap I=0$; hence Proposition 3 implies $A \leqq Z_{r}(R)$. Thus we have $A=0$, which shows that $I$ is essential in $J_{R}$. Since $I$ is essentially closed in $R_{R}$ by Lemma 1 , we must have $I=J$. Therefore, $I$ is indeed a semiprime ideal of $R$.

Conversely, assume that $I$ is a semiprime ideal which is essentially closed in $R_{R}$, and set $\bar{R}=R / I$. According to Lemma 1 , it suffices to show that $\bar{R}_{\bar{R}}$ is non-singular. Let $x+I \in Z_{r}(\bar{R})$, and set $A=\{a \in R \mid x a \in I\}$. Then $A$ is an essential right ideal of $R$, and $r_{\bar{R}}(x+I)=A / I$. By Proposition 4, $A$ contains an ideal $H$ essential in $R_{R}$. Set $\bar{H}=(H+I) / I$. Since $I$ is essentially closed in $R_{R}$, the essentiality of $H$ implies that $\bar{H}$ is essential in $\bar{R}_{\bar{R}}$. Now, $\left(l_{\bar{R}}(\bar{H}) \cap \bar{H}\right)^{2} \leqq$ $l_{\bar{R}}(\bar{H}) \bar{H}=0$; hence we see that $l_{\bar{R}}(\bar{H})=0$, because $\bar{R}$ is a semiprime ring. Thus we have $x+I \in l_{\bar{R}}(\bar{H})=0$, from which we conclude that $\bar{R}_{\bar{R}}$ is non-singular.

Immediately, Corollary 5 implies the following result which is a generalization of [2, Proposition 2.5] and [3, Theorem 3.3].

Corollary 6. A right GFC ring is right non-singular if and only if it is a semiprime ring.

By $[8$, Proposition $V I, 6.2]$, we have $G(R)=\left\{x \in R \mid x+Z_{r}(R) \in Z_{r}\left(R / Z_{r}(R)\right)\right\}$. Thus [3, Theorem 5.1] shows that if $R$ is right FPF then $G(R)$ is a direct summand of $R$ as a right ideal and $R / G(R)$ is a non-singular right FPF ring. More generally we have the following result.

Proposition 7. Assume that $R$ is right FPF, and let $I$ be an ideal which is $G$ closed in $R_{R}$. Then

(1) $I$ is a direct summand of $R_{R}$. 
(2) $R / I$ is a right and left non-singular right FPF ring.

Proof. (1) Choose a right ideal $A$ of $R$ maximal with respect to $A \cap I=0$. Then $R / A \oplus R / I$ is finitely generated faithful; hence by assumption, $R=\operatorname{Tr}_{R}$ $(R / A \oplus R / I)=\operatorname{Tr}_{R}(R / A)+\operatorname{Tr}_{R}(R / I)=l_{R}(A) R+l_{R}(I)$. Set $\bar{R}=R / I$ and $\bar{A}=(A \oplus$ $I) / I$. Then, observing that $I$ is essentially closed in $R_{R}$ by Lemma 1 and that $A \oplus I$ is essential in $R$, we see that $\bar{A}$ is an essential right ideal of $\bar{R}$. Since $\bar{A} \leqq r_{\bar{R}}(x+I)$ for every $x \in l_{R}(A)$, it follows from the essentiality of $\bar{A}$ and Lemma 1 that $l_{R}(A) \leqq I$. Thus we obtain $R=I+l_{R}(I)$. Writing $1=a+b$ where $a \in I$ and $b \in l_{R}(I)$, we see that $a$ is an idempotent of $R$ and $I=a R$. Consequently, $I$ is a direct summand of $R_{R}$.

(2) Let $M$ be a finitely generated faithful right $\bar{R}$-module and set $X=I \oplus$ $M$. Since $r_{R}(X)=r_{R}(I) \cap r_{R}(M)=r_{R}(I) \cap I$, we see from (1) that $r_{R}(X)=0$; hence $X$ is a finitely generated faithful right $R$-module. Thus by assumption, in particular, $X$ generates $R / I$, while (1) says $\operatorname{Hom}_{R}\left(I_{R},(R / I)_{R}\right)=0$. It then follows that $M$ generates $R / I$ as a right $R$-module and so does as a right $(R / I)$ module. Therefore we conclude that $R / I$ is a right FPF ring. Moreover, Lemma 1 and [3, Theorem 3.6] imply that $R / I$ is a right and left non-singular ring.

As consequences of Proposition 7, we obtain the following results.

Corollary 8. If $R$ is right FPF then every $G$-closed right ideal of $R$ is a right annihilator ideal of $R$.

Proof. Given any $G$-closed right ideal $A$ of $R$, choose a right ideal $C$ of $R$ maximal with respect to $C \leqq r_{R} l_{R}(A)$ and $A \cap C=0$. If $C=0$ then we see from the $G$-closedness of $A$ that $A=r_{R} l_{R}(A)$, which completes the proof. Thus it is enough to show that $C=0$.

Choose a right ideal $B$ of $R$ maximal with respect to $A \leqq B$ and $B \cap C=0$. Since $C$ is non-singular and $R / B$ is an essential extension of $C$, we see that $B$ is $G$-closed in $R$; hence $G(R) \leqq B$. On the other hand, observing that $B \oplus C$ is essential in $R$ and that it is contained in $r_{R} l_{R}(B)$, we see that $r_{R} l_{R}(B)$ is essential in $R$; hence $l_{R}(B) \leqq G(R)$. Thus we have $l_{R}(B) \leqq r_{R}(R / B)$, which implies $\operatorname{Tr}_{R}(R / B) \leqq r_{R}(R / B)$. Since $B$ is $G$-closed in $R_{R}$ and hence so is $r_{R}(R / B)$, Proposition 7 shows that $R$ is a direct sum of $r_{R}(R / B)$ and a right ideal of $R$ generated by $R / B$; hence in particular, we have $R=r_{R}(R / B)+\operatorname{Tr}_{R}(R / B)$. It then follows $R=r_{R}(R / B)$, that is, $B=R$, from which $C$ must be zero, as desired.

Corollary 9. Assume that $R$ is right FPF. If $M$ is a finitely generated non-singular right $R$-module with finite Goldie dimension then $\operatorname{End}_{R}(M)$ is a twosided order in a semisimple ring.

Proof. Since $r_{R}(M)$ is $G$-closed in $R_{R}$ and $M$ is non-singular as a right 
$R / r_{R}(M)$-module, without loss of generality we may assume by Proposition 7 that $M$ is faithful and $R$ is non-singular. It then follows that $R$ is isomorphic to a direct summand of a finite direct sum of copies of $M$; hence $R_{R}$ has finite Goldie dimension, because $M$ has finite Goldie dimension. Now, we see from Corollary 6 and [3, Corollary $3.16 \mathrm{C}$ ] that $R$ is a semiprime right and left Goldie ring. Therefore, [6, Theorems 2.2.15 and 2.2.17] show that $\operatorname{End}_{R}(M)$ is a two-sided order in a semisimple ring.

Let $\tau$ be a hereditary torsion theory for mod- $R$. Then $\tau$ is stable if the $\tau-$ torsion class is closed under injective envelopes, and $L(\tau)$ is bounded if it contains a cofinal subset consisting of ideals of $R$. We note from [8, Proposition VI, 7.3] that $G$ is stable, and from [8, Chapter VI, Section 6.3] that if $R$ is right non-singular then $L(G)$ consists of all the essential right ideals of $R$; hence $R$ is right essentially bounded if and only if $L(G)$ is bounded.

To provide a characterization of right FPF rings, we need the following result.

Lemma 10. Let $\tau$ be a stable hereditary torsion theory for mod- $R$ such that $L(\tau)$ is bounded. For a finitely generated right $R$-module $M$, the following conditions are equivalent:

(1) $r_{R}(M) \leqq \tau(R)$.

(2) $r_{R}(M / \tau(M))=\tau(R)$.

Proof. First we shall show $r_{R}(\tau(M)) \in L(\tau)$. To this end, choose a submodule $N$ of $M$ maximal with respect to $\tau(M) \cap N=0$. Observing that $\tau$ is stable and that $M / N$ is an essential extension of $\tau(M)$, we see that $M / N$ is $\tau$ torsion. Since $M$ is finitely generated, $M / N=x_{1} R+\cdots+x_{n} R$ for a finite number of elements $x_{1}, \cdots, x_{n} \in M / N$. Further, since $M / N$ is $\tau$-torsion and $L(\tau)$ is bounded, there exist ideals $I_{i} \in L(\tau)(i=1, \cdots, n)$ such that $I_{i} \leqq r_{R}\left(x_{i}\right)$ for each $i$. We then see that $\bigcap_{i=1}^{n} I_{i} \in L(\tau)$ and $\bigcap_{i=1}^{n} I_{i} \leqq r_{R}(M / N) \leqq r_{R}(\tau(M))$, from which we conclude $r_{R}(\tau(M)) \in L(\tau)$.

(1) $\Rightarrow(2)$. Since $L_{\tau}(R)=0$ for every $\tau$-torsionfree right $R$-module $L$, we always have $\tau(R) \leqq r_{R}(M / \tau(M))$. Conversely, according to (1), we have $r_{R}$ $(M / \tau(M)) r_{R}(\tau(M)) \leqq r_{R}(M) \leqq \tau(R)$. Now, noting that $R / \tau(R)$ is $\tau$-torsionfree and that $r_{R}(\tau(M)) \in L(\tau)$ as is seen above, we see $r_{R}(M / \tau(M)) \leqq \tau(R)$. Thus we obtain $r_{R}(M / \tau(M))=\tau(R)$.

$(2) \Rightarrow(1)$ is clear.

In [7] Kobayashi has provided a characterization of non-singular right FPF rings. Now we state a characterization of right FPF rings, a part of which is an extension of [7, Theorem 1].

Theorem 11. The following conditions on $R$ are equivalent: 
(1) $R$ is right FPF.

(2) (i) For every finitely generated non-singular right $R$-module $M, R$ is a direct sum of $r_{R}(M)$ and a right ideal generated by $M$.

(ii) $L(G)$ is bounded.

(iii) Every finitely generated faithful right $R$-module generates $G(R)$.

(3) (i) For every finitely generated right ideal $A$ of $R$ such that $r_{R}(A)$ is $G$-closed in $R_{R}, R$ is a direct sum of $r_{R}(A)$ and a right ideal generated by $A$.

(ii) $L(G)$ is bounded.

(iii) For every finitely generated faithful right $R$-module $M$ such that $G(M)$ is a direct summand of $M, G(M)$ generates $G(R)$.

(iv) Every finitely generated non-singular right $R$-module can be embedded into a free right $R$-module.

Proof. (1) $\Rightarrow(2)$. (2) (i) follows from Proposition 7, and (2) (iii) is clear.

To show (2) (ii), let $A \in L(G)$ and set $I=r_{R}(R / A), G(R / I)=J / I$ and $M=$ $(R / A) \oplus J$. Then $J$ is an ideal which is $G$-closed in $R_{R}$. It follows from Proposition 7 that $J=e R$ for some idempotent $e$ of $R$ and $r_{R}(M)=I \cap r_{R}(J) \leqq e R \cap$ $(1-e) R=0$; hence $M$ is finitely generated faithful. According to (1), $M$ is a generator of mod- $R$, in particular, $M$ generates $(1-e) R$. However, $\operatorname{Hom}_{R}(M$, $(1-e) R)=\operatorname{Hom}_{R}(R / A,(1-e) R) \oplus \operatorname{Hom}_{R}(J,(1-e) R)=0 \oplus 0=0$, from which we see $e=1$. Thus $G(R / I)=R / I$, that is, $r_{R}(R / A)=I \in L(G)$. Therefore, $L(G)$ is bounded.

$(2) \Rightarrow(3)$. First we shall assume (2) and show the following

Claim 1. For every ideal I which is $G$-closed in $R_{R}, R / I$ is a right FPF ring.

Set $\bar{R}=R / I$ and let $\bar{G}$ denote the Goldie torsion theory for mod- $\bar{R}$. Since $\bar{R}$ is a right non-singular ring by Lemma $1, L(\bar{G})$ consists of all the essential right ideals of $\bar{R}$. First we show that $L(\bar{G})$ is bounded. Let $\bar{A}=A / I \in L(\bar{G})$. Then $A$ is essential in $R$; hence $A \in L(G)$. According to (2) (ii), there exists an ideal $J$ of $R$ such that $J \leqq A$ and $J \in L(G)$. If $\bar{B}=B / I$ is a right ideal of $\bar{R}$ such that $\bar{J} \cap \bar{B}=0$ where $\bar{J}=(J+I) / I$, then $\bar{B} \cdot \bar{J}=\bar{B} \cap \bar{J}=0$, that is, $B \cdot J \leqq I$, from which we have $B \leqq I$, because $(R / I)_{R}$ is non-singular. Thus $J$ is essential in $\bar{R}_{\bar{R}}$, which shows that $L(\bar{G})$ is bounded. Now, we turn to the proof of Claim 1. Let $M$ be a finitely generated faithful right $\bar{R}$-module. We must show that $M$ is a generator of $\bmod -\bar{R}$. Since $M / \bar{G}(M)$ is a faithful right $\bar{R}$-module by Lemma 10 and $M$ obviously generates $M / \bar{G}(M)$, we may assume that $M_{\bar{R}}$ is non-singular; hence it is non-singular as an $R$-module, also. According to (2) (i), $M$ generates $R / r_{R}(M)=\bar{R}_{\bar{R}}$, from which we conclude that $\bar{R}$ is right FPF. This completes the proof of Claim 1.

(3) (i) is immediate from (2) (i) and Claim 1.

To show (3) (iii), let $M$ be a finitely generated faithful right $R$-module. By (2) (iii), we obtain an exact sequence $M^{(n)} \rightarrow G(R) \rightarrow 0$, and further it splits, be- 
cause $G(R)_{R}$ is projective by (2) (i). Thus we may assume that $M^{(n)}=G(R) \oplus N$ for some integer $n$ and some submodule $N$ of $M^{(n)}$. It now follows $G(M)^{(n)}=$ $G\left(M^{(n)}\right)=G(R) \oplus G(N)$, from which we see that $G(M)$ generates $G(R)$.

Finally, to show (3) (iv), let $M$ be a finitely generated non-singular right $R$-module. Then $M$ is finitely generated non-singular as a right $R / r_{R}(M)$ module, while (2) (i) implies $R=r_{R}(M) \oplus A$ for some right ideal $A$ of $R$. It then follows from Claim 1, [3, Theorem 3.12] and [5, Theorem 5.17] that $M$ is embedded into $\left(R / r_{R}(M)\right)^{(n)} \cong A^{(n)} \leqq R_{R}^{(n)}$ for some integer $n$.

$(3) \Rightarrow(1)$. First we shall assume (3) and show the following

Claim 2. (1) $G(R)$ is a direct summand of $R$ as a right ideal.

(2) For every finitely generated non-singular right $R$-module $M$ such that $r_{R}(M)=G(R), M$ generates $R / G(R)$.

Let $M$ be a finitely generated non-singular right $R$-module such that $r_{R}(M)$ $=G(R)$. By (3) (iv), we obtain an exact sequence $0 \rightarrow M \stackrel{f}{\rightarrow} R^{(n)}$ for some integer $n$. Let $p_{i}: R^{(n)} \rightarrow R$ be the $i$-th projection $(i=1, \cdots, n)$ and set $A=\sum_{i=1}^{n} p_{i} f(M)$. Then $A$ is a finitely generated right ideal of $R$ and $r_{R}(A)=G(R)$; hence (3) (i) says that $R$ is a direct sum of $G(R)$ and a right ideal $B$ generated by $A$. Since $M$ obviously generates $A$, it also generates $B \cong R / G(R)$, which completes the proof of Claim 2.

To show that $R$ is right FPF, let $M$ be a finitely generated faithful right $R$-module, and choose a submodule $N$ of $M$ maximal with respect to $N \cap G(M)$ $=0$. Since $M / N$ is an essential extension of $G(M)$, it is $G$-torsion; hence setting $X=M / G(M) \oplus M / N$, we see that $G(X)=M / N$ and that $X$ is finitely generated faithful. It now follows from (3) (iii) that $G(X)=M / N$ generates $G(R)$. On the other hand, by (3) (ii) and Lemma 10, we have $r_{R}(X / G(X))=$ $G(R)$; hence Claim 2(2) shows that $X / G(X) \simeq M / G(M)$ generates $R / G(R)$. Since $M$ obviously generates both $M / N$ and $M / G(M)$ and since $R \cong G(R) \oplus(R / G(R))$ by Claim 2(1), $M$ generates $R$. This completes the proof of the theorem.

Assume that $R$ is non-singular right FPF and let $M$ be a finitely generated non-singular right $R$-module. It then follows from Theorem 11 that $R=r_{R}(M)$ $\oplus A$ where $A$ is a right ideal of $R$ generated by $M$. Since $R$ is a semiprime ring by Corollary 6 , we see $\operatorname{Hom}_{R}\left(M, r_{R}(M)\right)=0$, which implies $A=\operatorname{Tr}_{R}(M)$. Thus $R=r_{R}(M) \oplus \operatorname{Tr}_{R}(M)$ as ideals. Therefore, as a consequence of Theorem 11 , we obtain the following result, in which $(1) \Leftrightarrow(3)$ is due to [7, Theorem 1] (c.f. [5, Theorem 5.17]).

Corollary 12. For a right non-singular ring $R$, the following conditions are equivalent : 
(1) $R$ is right EPF.

(2) (i) For every finitely generated non-singular right $R$-module $M, R=$ $r_{R}(M) \oplus \operatorname{Tr}_{R}(M)$ as ideals.

(ii) $R$ is right essentially bounded.

(3) (i) For every finitely generated right ideal $A$ of $R, R=r_{R}(A) \oplus \operatorname{Tr}_{R}(A)$ as ideals.

(ii) $R$ is right essentially bounded.

(iii) Every finitely generated non-singular right $R$-module can be embedded into a free right $R$-module.

We call a ring homomorphism $\psi: R \rightarrow S$ a flat epimorphism if it is an epimorphism in the category of rings (or equivalently, the natural homomorphism $S \otimes_{R} S \rightarrow S$ is an isomorphism by [8, Chapter XI, Section 1]) and $S$ is flat as a right $R$-module. We note that if both $\psi: R \rightarrow S$ and $\zeta: S \rightarrow T$ are flat epimorphisms then so is $\zeta \psi: R \rightarrow T$. For the Goldie torsion theory $G$ for mod- $R$, we denote by $Q_{G}$ the ring of quotients of $R$ with respect to $G$ and by $\varphi: R \rightarrow Q_{G}$ the canonical ring homomorphism.

Now assume that $R$ is right FPF, and set $Q=Q_{G}$. Since $\varphi(R) \cong R / G(R)$ is projective as a right $R$-module by Proposition 7, we see that $\phi: R \rightarrow \varphi(R)$ is a flat epimorphism. We also note from [8, Chapter IX, Sections 1 and 2] that $\operatorname{Hom}_{R}\left((Q / \varphi(R))_{R}, Q_{R}\right)=0$ and $Q_{R}$ is injective and non-singular, and from Theorem 11 that if $x \in Q$ then $\varphi(R)+x \varphi(R)$ can be embedded into $R^{(n)}$ (in fact, into $\left.\varphi(R)^{(n)}\right)$ for some integer $n$. Now, following the same argument as in the proof of $(a) \Rightarrow(b)$ of [5, Theorem 5.17], we see that if $x \in Q$ and $J=\{\phi(r) \in \varphi(R) \mid \varphi(r) x$ $\in \varphi(R)\}$ then $Q J=Q$. It then follows from [5, Theorem 3.9] that the inclusion map $\varphi(R) \rightarrow Q$ is a flat epimorphism. Thus we have the following result.

Corollary 13. If $R$ is right EPF then $\varphi: R \rightarrow Q_{G}$ is a flat epimorphism.

Finally, we present examples to illustrate Theorem 11.

EXAMPLE 1. There exists a ring satisfying the conditions (2) (ii) and (iii) ((3) (ii), (iii) and (iv)) of Theorem 11, but not FPF.

Proof. Set $R=\{(x, y) \in Z \times Z \mid x \equiv y(\bmod 2)\}$ where $Z$ is the ring of integers. Then $R$ is a commutative semiprime Noetherian ring; hence it satisfies (2) (ii) and (iii) ((3) (ii), (iii) and (iv)) of Theorem 11.

Now, set $A=(2,0) R \oplus(0,2) R$. Then $A$ is finitely generated faithful, but $\operatorname{Tr}_{R}(A)=A \neq R$; hence $R$ is not FPF.

EXAMPLE 2. There exists a ring satisfying the conditions (2) (i) and (iii) ((3) (i), (iii) and (iv)) of Theorem 11, but not FPF.

Proof. Let $R$ be a simple principal ideal domain but not a skew field (c.f. 
[6, Proposition 1.3.8]). Then $R$ satisfies the conditions (2) (i) and (iii) ((3)(i), (iii) and (iv)) of Theorem 11, while $L(G)$ is not bounded; hence $R$ is not FPF by Theorem 11.

EXAMPLE 3. There exists a ring satisfying the conditions (2) (i) and (ii) ((3) (i), (ii) and (iv)) of Theorem 11, but not FPF.

Proof. Let $F$ be a field and set $R=\left[\begin{array}{ll}F & F[x] /\left(x^{2}\right) \\ 0 & F[x] /\left(x^{2}\right)\end{array}\right]$. Then $Z_{r}(R)=$ $\left[\begin{array}{ll}0 & (x) /\left(x^{2}\right) \\ 0 & (x) /\left(x^{2}\right)\end{array}\right]$ and it is essential in $R_{R}$; hence $R_{R}$ is $G$-torsion, from which it trivially satisfies the conditions (2) (i) and (ii) ((3) (i), (ii) and (iv)) of Theorem 11.

Now, set $A=\left[\begin{array}{cc}F & F[x] /\left(x^{2}\right) \\ 0 & 0\end{array}\right]$. Then $A$ is a faithful right ideal generated by $\left[\begin{array}{ll}1 & 0 \\ 0 & 0\end{array}\right]$, but $\operatorname{Tr}_{R}(A)=A \neq R$; hence $R$ is not right FPF.

EXAMPLE 4. There exists a ring satisfying the conditions (3) (i), (ii) and (iii) of Theorem 11, but not FPF.

Proof. Let $F$ be a field, and set $F=F_{i}$ for $i=1,2, \cdots$, and $R=\left\{x=\left(x_{i}\right) \in\right.$ $\prod_{i=1}^{\infty} F_{i} \mid$ there exists an integer $n$ such that $x_{n}=x_{i}$ for all but finitely many $\left.i\right\}$. Then $R$ is a commutative von Neumann regular ring which is not self-injective, and it then satisfies the conditions (3) (i), (ii) and (iii) of Theorem 11. But, [5, Theorem 3.12] and Theorem 11 imply that $R$ is not FPF.

Acknowledgement. The author would like to express his thanks to Professor Y. Kurata for his valuable advice in preparing this paper,

\section{References}

[1] F.W. Anderson and K.R. Fuller: Rings and Categories of Modules, SpringerVerlag, New York-Heidelberg-Berlin, 1973.

[2] G.F. Birkenmeier: A generalization of FPF rings, Comm. Algebra 17 (1989), 855884.

[3] C. Faith and S. Page: FPF Ring Theory, London Math. Soc. Lecture Note Series 88, Cambridge University Press, Cambridge, 1984.

[4] T.G. Faticoni: FPF rings I: The Noetherian case, Comm. Algebra 13 (1985), 2119-2136.

[5] K.R. Goodearl: Ring Theory, Marcel Dekker, New York-Basel, 1976.

[6] A.V. Jategaonkar: Localization in Noetherian Rings, London Math. Soc. Lecture Note Series 98, Cambridge University Press, Cambridge, 1986.

[7] S. Kobayashi: On non-singular FPF rings I, Osaka J. Math. 22 (1985), 787-795.

[8] B. Stenström: Rings of Quotients, Springer-Verlag, Berlin-Heidelberg-New York, 1975. 
H. Yoshimura

Department of Mathematics

Yamaguchi University

Yoshida, Yamaguchi 753, Japan 